function of the apparent magnitude and a parameter which is derived by least squares from the radial velocities and proper motions of the whole group of observed Class B stars. His value is 0"0071 ( $=458$ light-years), which, since it depends immediately upon the parameter, cannot be appreciably affected by the small correction here found for the velocity of the binary system.

These three values of parallax, all of which may for the present be called hypothetical, show a remarkable agreement. The three methods of investigation appear to be entirely independent, employing the same data but a different law or relation of certain of the quantities. They have, however, a common basis in that they assume some sort of interrelation among these stars.

\section{G. F. PADDOCK.}

December', 1916.

\title{
NOTE ON THE IDENTIFICATION OF TWO MORE LINES IN THE SPECTRUM $\mathrm{OF} v_{4} \operatorname{ERIDANI}$
}

The writer of the Astronomical Column in the issue of Nature for January 13, 1916, volume 96, page 547 , has kindly pointed out identifications for two unidentified lines of the spectrum of $v_{4}$ Eridani, which are given in Table III, Lick Observatory Bulletin 274, volume 8, page 172. His references are: For line 4282.7A, Baxandall's Enhanced Lines of Manganese in the Spectrum of a Aindromedae, Mon. Not., 74, 252 , 1914 ; for line 4416.9A, Fowler's Enhanced Lines of Iron . . ., Mon. Not., 67, 156, 1906. These two lists escaped notice in the compilation of Table III for $v_{4}$ Eridani. All the lines noted as manganese in Table
III, together with line 4282.7A, are given in Baxandall's list, in which the wavelength of the latter line is $4282.65 \mathrm{~A}$ as found in the laboratory and $4282.87 \mathrm{~A}$ in the spectrum of a Andromedae. Fowler finds the enhanced iron line $4416.98 \mathrm{~A}$ to be given by the positive pole of the arc, and notes that it is masked by an air line in the spark.

\section{G. F. PADDOCK.}

January, 1917.

Issued January 16, 1917. 